\title{
Enhanced Ad Hoc On-demand Distance Vector (EAODV) Routing Protocol with Route Distribution
}

\author{
Bong Chan $\mathrm{Kim}^{1}$, Hwang Soo Lee ${ }^{1}$, and Joong Soo $\mathrm{Ma}^{2}$ \\ Dept. Electrical Engineering and Computer Science, KAIST, Republic of Korea ${ }^{1}$ \\ School of Engineering, ICU, Republic of Korea ${ }^{2}$ \\ Email: eeman@mcl.kaist.ac.kr
}

\begin{abstract}
Ad hoc on-demand distance vector (AODV) routing protocol is a representative ad hoc routing protocol based on an on-demand approach. In the route discovery procedure of AODV, a source broadcasts a route request (RREQ) packet to find a route to a destination. If an intermediate node has a valid route to the destination in its own route table, the intermediate node can reply to the RREQ with a route reply (RREP) packet and discard the RREQ packet. We term this mechanism as the route cache mechanism. The route cache mechanism has the merits of a short route setup time, a high route setup probability, and a small propagation range of RREQ packets. However, the route cache mechanism has the demerit of route concentration on a few mobile nodes. In this paper, we propose an enhanced AODV (EAODV) routing protocol with a selective route cache mechanism based on a source/destination pair in the route discovery procedure. By the selective route cache mechanism, EAODV can maintain the merits of the route cache mechanism and solve its demerit simultaneously. We compare the performances of the conventional AODV and EAODV in multihop access environment, where several mobile nodes send data packets to a fixed node such as an access point (AP), via ns-2 simulation. Simulation results show that EAODV outperforms the conventional AODV in terms of the packet delivery ratio, the average end-to-end delay, and the normalized routing overhead because EAODV can get the effect of route distribution in the route discovery procedure by the selective route cache mechanism based on a source/destination pair.
\end{abstract}

Keywords: Ad hoc network, AODV, route distribution

\section{INTRODUCTION}

A mobile ad hoc network (MANET) [1] is a collection of mobile nodes which are formed temporarily without any aid of an established infrastructure. Mobile ad hoc networks have the following features: first, movement direction and speed of mobile nodes can not be predicted. Second, since power supply of mobile nodes is based on battery, available power is limited. Lastly, since a shared wireless medium is used as a medium for packet transmission, available bandwidth is limited.

To overcome the limitations of such a complex environment, many ad hoc routing protocols $[2][3][4][5][6][7][8]$ have been proposed for MANET. Initial ad hoc routing protocol such as destination sequenced distance vector (DSDV) [2] is based on a table-driven approach. In ad hoc routing protocols based on a table-driven approach, mobile nodes should periodically exchange their own route information with neighbor nodes for a route table update. Due to considerable routing overhead, however, ad hoc routing protocols based on a table-driven approach are not desirable to ad hoc networks in terms of power and bandwidth efficiency.

To solve limitations of the table-driven approach, an ondemand approach is applied to ad hoc routing protocol design. AODV [3] is a representative ad hoc routing protocol based on an on-demand approach. In the route discovery procedure of AODV, a source broadcasts an RREQ packet to find a route to a destination. If an intermediate node has a valid route to the destination in its own route table, the intermediate node can reply to the RREQ with an RREP packet and discard the RREQ packet. We term this mechanism as the route cache mechanism. The route cache mechanism has the merits of a short route setup time, a high route setup probability, and a small propagation range of RREQ packets. However, the route cache mechanism has the demerit of route concentration on a few mobile nodes.

In this paper, we propose an enhanced AODV (EAODV) with a selective route cache mechanism based on a source/destination pair. EAODV maintains the merits of the route cache mechanism and solves its demerit by the selective route cache mechanism in the route discovery procedure. Following this INTRODUCTION, the features of the route cache mechanism in the route discovery procedure of AODV is explained in detail in Section II. In Section III, we propose EAODV with the selective route cache mechanism. Simulation environments for the performance evaluation of EAODV are described in Section IV. In Section V, we show simulation results. Finally, we make a conclusion in Section VI.

\section{Features Of Route Cache Mechanism}

In AODV, the route cache mechanism means that if intermediate nodes have a valid route to a received RREQ's destination, they can reply to the RREQ with an RREP packet and discard the RREQ. In this Section, we explain the features of the route cache mechanism in the route discovery procedure of AODV.

\section{A. Route Cache Mechanism and Routing Overhead}

By the route cache mechanism, intermediate nodes with a valid route to a received RREQ's destination can reply to the RREQ with an RREP and discard the RREQ. Thus, the propagation range of RREQ packets can be reduced. However, the route cache mechanism generates many RREP packets by several intermediate nodes' replies. 


\section{B. Route Cache Mechanism and Route Setup Time}

If a source initiates the route discovery procedure to find a route to a destination, it can receive replies from intermediate nodes by the route cache mechanism. Thus, the route setup time to a destination can be reduced.

\section{Route Cache Mechanism and Route Setup Probability}

We define the following notations:

- $\quad \mathrm{p}$ : probability to fail to transmit a packet between a pair of nodes.

- $\mathrm{n} 1$ : hop counts between a source and a destination.

- n2: hop counts between a source and the closest intermediate node with a route to a destination.

If AODV does not use the route cache mechanism in the route discovery procedure,

Route setup probability $(\mathrm{p} 1)=(1-\mathrm{p})^{2 * \mathrm{n} 1}$

If AODV uses the route cache mechanism in the route discovery procedure,

Route setup probability $(\mathrm{p} 2)=(1-\mathrm{p})^{2 * \mathrm{n} 2}$

Because of $\mathrm{n} 1>\mathrm{n} 2$, AODV with the route cache mechanism has better performance than AODV without the route cache mechanism in the term of the route setup probability.

\section{Route Cache Mechanism and Route Concentration}

Figs. 1a and $1 \mathrm{~b}$ show the effect of route concentration on a few nodes by the route cache mechanism in the route discovery procedure of AODV. In the figures, dotted line between a pair of nodes means that they are within transmission range each other. In the Fig. 1a, node 6 establishes the route to node 1, which is indicated by the bold solid line with arrow, to send data packets. After route establishment from node 6 to node 1, if node 7 broadcasts an RREQ with TTL $=1$ for a route discovery to node 1 , since node 6 has the already known route to node 1 by the previous route discovery procedure from node 6 to node 1 , node 6 will receive the RREQ and reply to node 7 with an RREP. Node 7 will receive the RREP from node 6 and start to send data packets over the established route, which is indicated by the bold dotted lines with arrow in Fig. 1b. As shown in Figs. 1a and $1 \mathrm{~b}$, the new route is established depending on the previously established routes by the route cache mechanism. Therefore, the route cache mechanism results in route concentration on a few nodes in the route discovery procedure.

\section{ENHANCED AODV (EAODV)}

In MANET, routes between sources and destinations are frequently broken down by wireless link failure and movement of nodes. So, a short route recovery time and a high route setup probability are important requirements in an ad hoc routing protocol design. A low routing overhead should be also considered to improve the efficiency of wireless resource. In this paper, we propose an enhanced AODV (EAODV) with a selective route cache mechanism. EAODV can maintain the

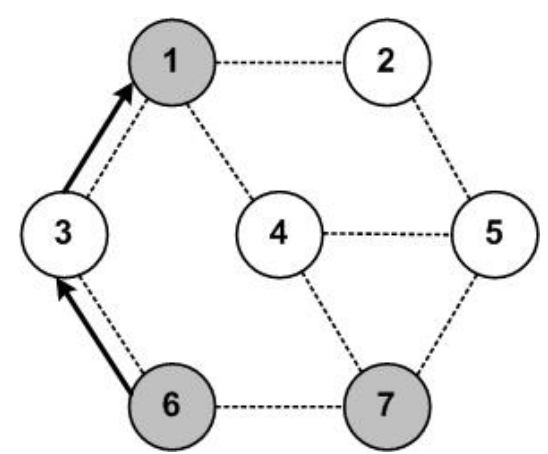

Fig. 1a - Route establishment from node 6 to node 1

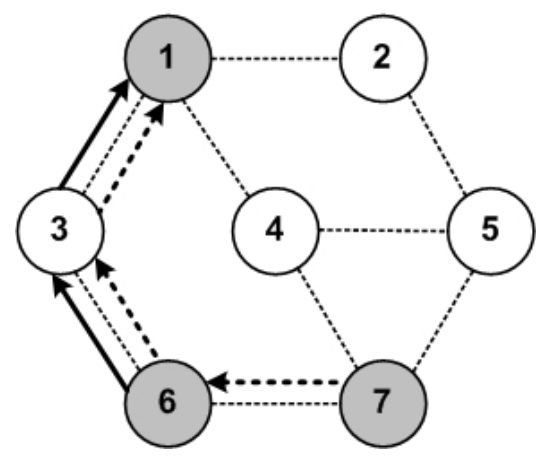

Fig. 1b - Route concentration on a few nodes by the route cache mechanism

merits of the route cache mechanism and solve its demerits by applying the selective route cache mechanism to the route discovery procedure. EAODV is also simple to implement and guarantees compatibility with the conventional AODV, which has already been standardized.

\section{A. Selective Route Cache Mechanism}

EAODV uses a selective route cache mechanism in the route discovery procedure. We define the selective route cache mechanism as follows: when a node has received an RREP, it stores a source/destination pair for a route established by the RREP in its route table entry. In the route discovery procedure, when an intermediate node has received an RREQ, if it has a valid route table entry to the RREQ's destination and the source/destination pair of the RREQ is the same as a source/destination pair stored in the route table entry, it replies to the RREQ with an RREP. Otherwise, the intermediate node broadcasts the RREQ.

\section{B. Route Discovery Procedure of EAODV}

In EAODV, the route discovery procedure is the same as the followings: in an RREQ reception, an intermediate node stores a reverse route to a source in its route table entry. If the intermediate node has a valid route table entry to the RREQ's destination and the source/destination pair of the RREQ is the same as the source/destination pair stored in the route table entry, it replies to the RREQ by the selective route cache 


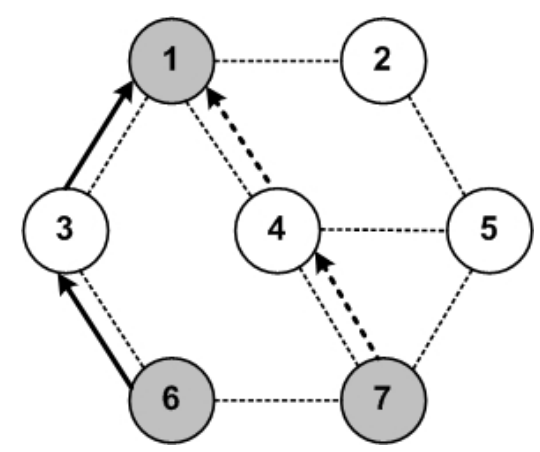

Fig. 2b - Route establishment from node 7 to node 1

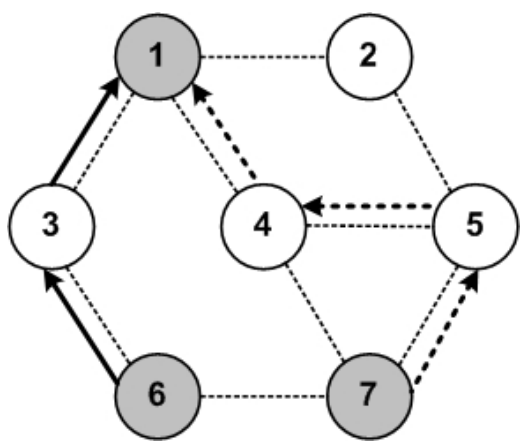

Fig. $2 \mathrm{~b}$ - Route establishment from node 7 to node 1 after the complete link failure between nodes 7 and 4

mechanism and discards it. Otherwise, the intermediate node broadcasts the RREQ packet. Once a destination receives an RREQ, it establishes a reverse route to a source and replies to the RREQ with an RREP. After the first reply, if the destination receives another RREQ with the same broadcast ID and destination address and the RREQ contains lower hop counts than the previously received RREQ, it replies to the RREQ again and discards it.

In an RREP reception, an intermediate node stores a forward route to a destination in a route table entry. In addition, the intermediate node stores a source/destination pair of the forward route in the route table entry for the selective route cache mechanism. The received RREP is sent to next hop over a previously established reverse route. Once a source receives an RREP, it establishes a forward route to a destination and discards the RREP. After the first RREP reception, if the source receives another RREP with the same broadcast ID and destination address and the RREP contain lower hop counts than the previously received RREP, it updates the route table entry to the destination and discards the RREP.

\section{Features of Selective Route Cachem Mechanism}

We examine features of the selective route cache mechanism. In Figs. $2 a$ and $2 b$, we assume that the route from node 6 to node 1 , which is indicated by bold solid lines with arrow in Fig. 2a, has already been established. If node 7 broadcasts an RREQ with TTL $=1$ to find a route to node 1 for data transmission, although node 6 has the route to node 1 by the previous route discovery procedure, node 6 does not reply to the received RREQ by the selective route cache mechanism. Since nodes 4 and 5 do not have a route to node 1 , they can not also reply to the RREQ. By the expanding ring search algorithm [3], node 7 broadcasts an RREQ with TTL=3 again. The RREQ will be propagated to node 1 and node 1 will reply to the RREQ. Thus, node 7 can obtain the route to node 1 , which is indicated by bold dotted lines with arrow in Fig. 2a, and there exists a chance of route distribution. We assume that the link between nodes 4 and 7 was broken down. By the expanding ring search algorithm, node 7 broadcasts an RREQ with $\mathrm{TTL}=2$ for a route recovery to node 1 . After temporary link failure, if the link between nodes 4 and 7 is recovered by itself, node 4 will receive the RREQ and reply by the selective route cache mechanism. Thus, node 7 can recover the same route as the previous one, which is indicated by bold dotted lines with arrow in Fig. 2a, within a short time. By the selective route cache mechanism, node 6 can not reply to the RREQ, although node 6 has a route to node 1 . If the link between node 4 and node 7 is broken down completely, node 4 will receive the RREQ through node 5 and reply with an RREP. Receiving the RREP from node 4 through node5, node 7 can recover the route to node 1 with partial feature of minimum hop counts within a short time. Bold dotted lines with arrow in Fig. 2 b represent the recovered route from node 7 to node 1. As explained above, the selective route cache mechanism can maintain merits of the route cache mechanism and solve its demerit simultaneously.

\section{SIMULATION ENVIRONMENTS}

To evaluate the performance of the selective route cache mechanism, we compare the performances of the conventional AODV with the route cache mechanism and EAODV with the selective route cache mechanism. The distributed coordination function (DCF) of IEEE 802.11 is used as the MAC protocol. The DCF uses request-to-send (RTS) and clear-to-send (CTS) control packets to reduce packet collisions resulting from the hidden node problem. For the radio model, we use the Lucent's WaveLAN parameters. WaveLAN has the transmission range of $250 \mathrm{~m}$ and the channel capacity of $2 \mathrm{Mb} / \mathrm{s}$. In both EAODV and AODV, RREQ and RERR are treated as broadcast packets and RREP and data packets are treated as unicast packets. Both AODV and EAODV use the link layer feedback signal from the MAC protocol to detect a link breakdown. They do not transmit hello messages periodically. [9]

\section{A. The Mobility and Traffic Models}

50 mobile nodes with speed between $0 \mathrm{~m} / \mathrm{s}$ and $20 \mathrm{~m} / \mathrm{s}$ move in a $1500 \mathrm{~m} * 300 \mathrm{~m}$ rectangular area according to the random waypoint model. An AP is located at $(750,150)$ for a $900 \mathrm{~s}$ simulation time. The duration of the pause time of mobile nodes is $300 \mathrm{~s}$. Thirty source nodes generate constant bit rate (CBR) traffic with the packet size of 512 bytes and the packet rate of 2packets/s as traffic source. The sources are randomly selected with uniform probability, but the destinations are selected in the way that $\alpha$ percentage of the selected destinations become the AP. We vary the AP traffic ratio $(\alpha)$ 
from $0 \%$ to $100 \%$ to verify the effect of route distribution by the selective route cache mechanism. We term this traffic model as the multi-hop access network scenario in our simulation.

\section{B. Performance Metrics}

Three performance metrics are used in our simulation:

Packet delivery ratio - The packet delivery ratio is the ratio of the total number of data packets received by destinations over the total number of data packets transmitted by sources.

Average end-to-end delay - The average end-to-end delay is the average of delays for all received data packets from the sources to destinations.

Normalized routing overhead - The normalized routing overhead is defined as the total number of routing control packets (RREQ, RREP, and RERR) normalized by the total number of the received data packets by the destinations.

\section{Simulation RESUlts}

\section{A. Packet Delivery Ratio}

In Fig. 3, we show the packet delivery ratios of AODV and EAODV in the multi-hop access network scenario. In this scenario, the packet delivery ratio of EAODV is usually better

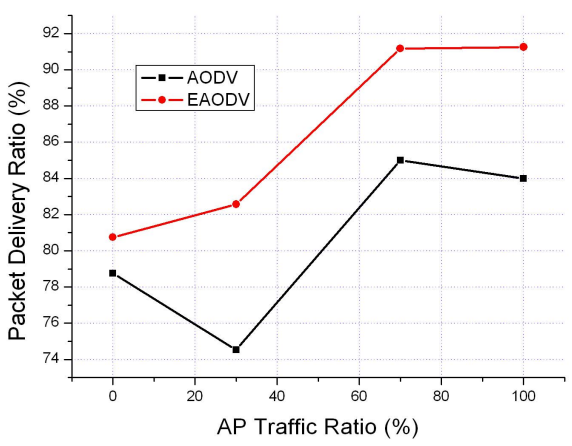

Fig. 3 - Packet delivery ratio in the multi-hop access network

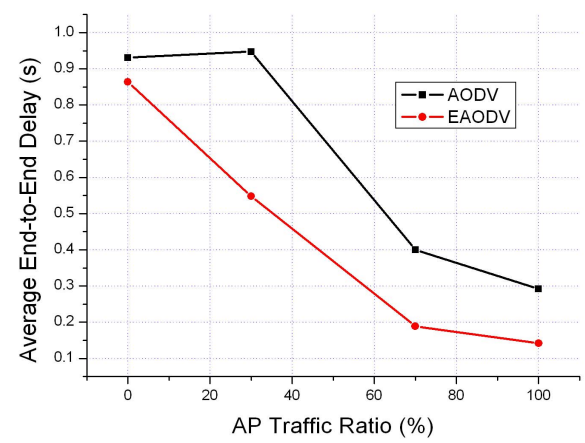

Fig. 4 - Average end-to-end delay in the multi-hop access network

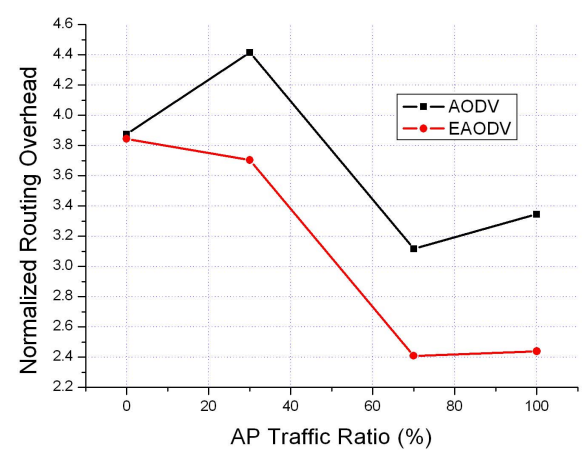

Fig. 5 - Normalized routing overhead in the multi-hop access network

than that of AODV regardless of the amount of traffic to AP, namely the degree of traffic concentration to AP. By the selective route cache mechanism, EAODV can select a fresh route with lower hop counts than AODV, which uses the route cache mechanism, in the route discovery procedure. Since the use of a fresh route reduces the probability of route breakdown, EAODV drops smaller amount of packets than AODV. EAODV has the effect of route distribution, whereas AODV has the effect of route concentration by the route cache mechanism. In EAODV, the effect of route distribution reduces the packet drop ratio due to buffer overflow in the heavily loaded node. Fig. 3 shows that as the amount of traffic to AP increases, EAODV has much better performance than AODV by the effect of route distribution in the term of the packet delivery ratio.

\section{B. Average End-to-End Delay}

In Fig. 4, we show the average end-to-end delays of AODV and EAODV in the multi-hop access network scenario. In this scenario, the average end-to-end delay of EAODV is usually better than that of AODV regardless of the amount of traffic to $\mathrm{AP}$. In the route discovery procedure of AODV, a new route is established depending on the already established routes by the route cache mechanism. This causes to select a route with long hop counts in the route discovery procedure and concentrate routes on a few mobile nodes. By the selective route cache mechanism in the route discovery procedure, however, EAODV can select a route with shorter hop counts than AODV and reduce queuing delay by distributing routes over the network. This causes to reduce the average end-to-end delay. We can verify the performance improvement of EAODV by the effect of route distribution in Fig. 4.

\section{Normalized Routing Overhead}

The normalized routing overheads of AODV and EAODV in the multi-hop access network scenario are shown in Fig. 5. In this scenario, the normalized routing overhead of EAODV is better than that of AODV regardless of the amount of traffic to AP, namely the degree of traffic concentration to AP. EAODV can reduce routing overhead occurring in the route discovery procedure by improving the performance of the packet delivery ratio. EAODV with the selective route cache mechanism has 


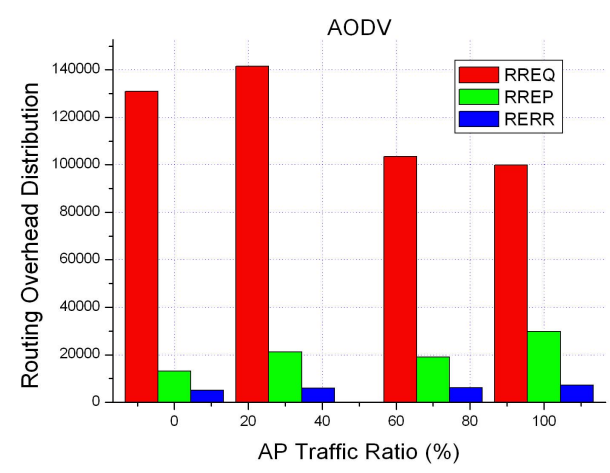

Fig. 6 - Distribution of routing overhead in the multi-hop access network (AODV)

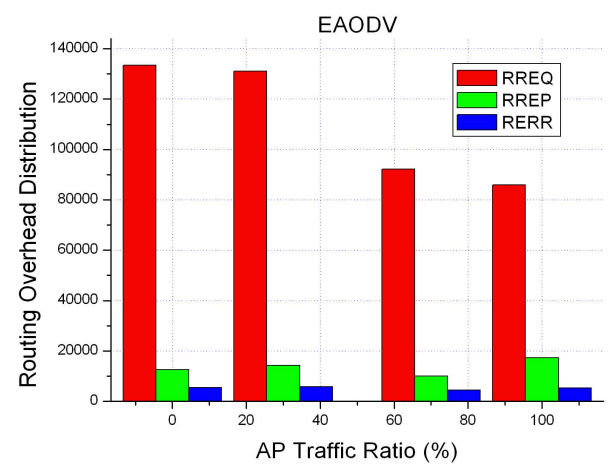

Fig. 7 - Distribution of routing overhead in the multi-hop access network (EAODV)

smaller number of RREP packets than AODV with the route cache mechanism. So, EAODV has better performance than AODV in the term of the normalized routing overhead. In AODV, if a mobile node initiates a route discovery procedure to the AP, since most of mobile nodes in the ad hoc network may have a route to the AP, many intermediate nodes will reply with an RREP packet by the route cache mechanism. By this RREP storm, the normalized routing overhead of AODV is much poorer than that of EAODV. In EAODV, RREP storm does not happen because EAODV uses the selective route cache mechanism in the route discovery procedure. Comparing Figs. 6 and 7, we can verify RREP storm of AODV.

\section{CONCLUSION}

In this paper, we proposed EAODV with the selective route cache mechanism. EAODV gets the merits of the route cache mechanism and solves its demerits by the selective route cache mechanism in the route discovery procedure. To evaluate the performance of EAODV, we compared the performances of EAODV and the conventional AODV via ns- 2 simulation. Simulation results showed that in the multi-hop access network scenario where most traffic is concentrated on an AP, EAODV has better performance than AODV by the effect of route distribution by the selective route cache mechanism in terms of the packet delivery ratio, the average end-to-end delay, and the normalized routing overhead.

\section{ACKNOWLEDGMENT}

This research was supported by the MIC (Ministry of Information and Communication), Korea, under the ITRC (Information Technology Research Center) support program supervised by the IITA (Institute of Information Technology Assessment)

\section{REFERENCES}

[1] Mobile Ad Hoc Network (MANET) [Online]. Available : http://www.ietf.org/html.charters/manet-charter.html.

[2] C. P. P. Bhagwat, "Highly Dynamic Destination-Sequenced Distance Vector Routing (DSDV) for Mobile Computers," in Proc. ACM SIGCOM'94, pp. 234-244, September 1994.

[3] C. Perkins, E. Royer, and S. Das, "Ad-hoc On-Demand Distance Vector (AODV) Routing," Network Working Group, RFC 3561, Jul. 2003.

[4] D. B. Johnson and D. A. Maltz. "Dynamic Source Routing in Ad Hoc Wireless Networks," in the book Mobile Computing, edited by Tomasz Imielinski and Hank Korth, chapter 5, pp. 153-181. Kluwer Academic Publishers, 1996.

[5] C. K Toh, "Ad Hoc Mobile Wireless Networks - Protocols and Systems," chapter 6, pp. 79-94, Prentice Hall PTR, 2002.

[6] S. Singh, M. Woo, and C. S. Raghavendra, "Power-Aware Routing in Mobile Ad Hoc Networks", in Proceedings of ACM/IEEE MobiCom'98 Conference, October 1998.

[7] R. Dube, C. D. Rais, K. Y. Wang, and S. K. Tripathi, "Signal stabilitybased adaptive routing (SSA) for ad hoc mobile networks", IEEE Pers. Commun., vol. 4, pp. 36-45, Feb. 1997.

[8] K. Wu and J. Harms, "Load-Sensitive Routing for Mobile Ad Hoc Networks," in Proc. Tenth International Conference on Computer Communications and Networks, pp. 540-546, Oct. 2001.

[9] C. E. Perkins, E. M. Royer, S. R. Das, and M. K. Marina, "Performance comparison of two on-demand routing protocols for ad hoc networks," IEEE Pers. Commun., vol. 8, pp. 16-28, Feb. 2001. 